\title{
Automated Tracking and Grasping of a Moving Object with a Robotic Hand-Eye System
}

\author{
Peter K. Allen, Member, IEEE, Aleksandar Timcenko, Student Member, IEEE, \\ Billibon Yoshimi, Student Member, IEEE, and Paul Michelman, Member, IEEE
}

\begin{abstract}
Most robotic grasping tasks assume a stationary or fixed object. In this paper, we explore the requirements for tracking and grasping a moving object. The focus of our work is to achieve a high level of interaction between a real-time vision system capable of tracking moving objects in 3-D and a robot arm with gripper that can be used to pick up a moving object. There is an interest in exploring the interplay of hand-eye coordination for dynamic grasping tasks such as grasping of parts on a moving conveyor system, assembly of articulated parts, or for grasping from a mobile robotic system. Coordination between an organism's sensing modalities and motor control system is a hallmark of intelligent behavior, and we are pursuing the goal of building an integrated sensing and actuation system that can operate in dynamic as opposed to static environments. The system we have built addresses three distinct problems in robotic hand-eye coordination for grasping moving objects: fast computation of 3-D motion parameters from vision, predictive control of a moving robotic arm to track a moving object, and interception and grasping. The system is able to operate at approximately human arm movement rates, and experimental results in which a moving model train is tracked is presented, stably grasped, and picked up by the system. The algorithms we have developed that relate sensing to actuation are quite general and applicable to a variety of complex robotic tasks that require visual feedback for arm and hand control.
\end{abstract}

\section{INTRODUCTION}

$\mathbf{T}$ HE focus of our work is to achieve a high level of interaction between a real-time vision system capable of tracking moving objects in 3-D and a robot arm equipped with a dextrous hand that can be used to intercept, grasp, and pick up a moving object. We are interested in exploring the interplay of hand-eye coordination for dynamic grasping tasks such as grasping of parts on a moving conveyor system, assembly of articulated parts, or for grasping from a mobile robotic system. Coordination between an organism's sensing modalities and motor control system is a hallmark of intelligent behavior, and we are pursuing the goal of building an integrated sensing and actuation system that can operate in dynamic as opposed to static environments.

There has been much research in robotics over the last few years that addresses either visual tracking of moving objects or generalized grasping problems. However, there have been

Manuscript received October 14, 1991; revised June 2, 1992. This work was supported in part by DARPA under Contract N00039-84-C-0165, by NSF under Grants DMC-86-05065, DCI-86-08845, CCR-86-12709, IRI-8657151, and IRI-88-1319 by North American Philips Laboratories, by Siemens Corporation, and by Rockwell Inc.

The authors are with the Department of Computer Science, Columbia University, New York, NY 10027

IEEE Log Number 9207358. few efforts that try to link the two problems. It is quite clear that complex robotic tasks such as automated assembly will need to have integrated systems that use visual feedback to plan, execute, and monitor grasping.

The system we have built addresses three distinct problems in robotic hand-eye coordination for grasping moving objects: fast computation of 3-D motion parameters from vision, predictive control of a moving robotic arm to track a moving object, and interception and grasping. The system is able to operate at approximately human arm movement rates, using visual feedback to track, intercept, stably grasp, and pick up a moving object. The algorithms we have developed that relate sensing to actuation are quite general and applicable to a variety of complex robotic tasks that require visual feedback for arm and hand control.

Our work also addresses a very fundamental and limiting problem that is inherent in building integrated sensing/actuation systems; integration of systems with different sampling and processing rates. Most complex robotic systems are actually amalgams of different processing devices, connected by a variety of methods. For example, our system consists of three separate computation systems: a parallel image processing computer; a host computer that filters, triangulates, and predicts 3-D position from the raw vision data; and a separate arm control system computer that performs inverse kinematic transformations and joint-level servoing. Each of these systems has its own sampling rate, noise characteristics, and processing delays, which need to be integrated to achieve smooth and stable real-time performance. In our case, this involves overcoming visual processing noise and delays with a predictive filter based upon a probabilistic analysis of the system noise characteristics. In addition, real-time arm control needs to be able to operate at fast servo rates regardless of whether new predictions of object position are available.

The system consists of two fixed cameras that can image a scene containing a moving object (Fig. 1). A PUMA-560 with a parallel jaw gripper attached is used to track and pick up the object as it moves (Fig. 2). The system operates as follows:

1) The imaging system performs a stereoscopic optic-flow calculation at each pixel in the image. From these opticflow fields, a motion energy profile is obtained that forms the basis for a triangulation that can recover the 3-D position of a moving object at video rates.

2) The 3-D position of the moving object computed by step 1 is initially smoothed to remove sensor noise, and a nonlinear filter is used to recover the correct 


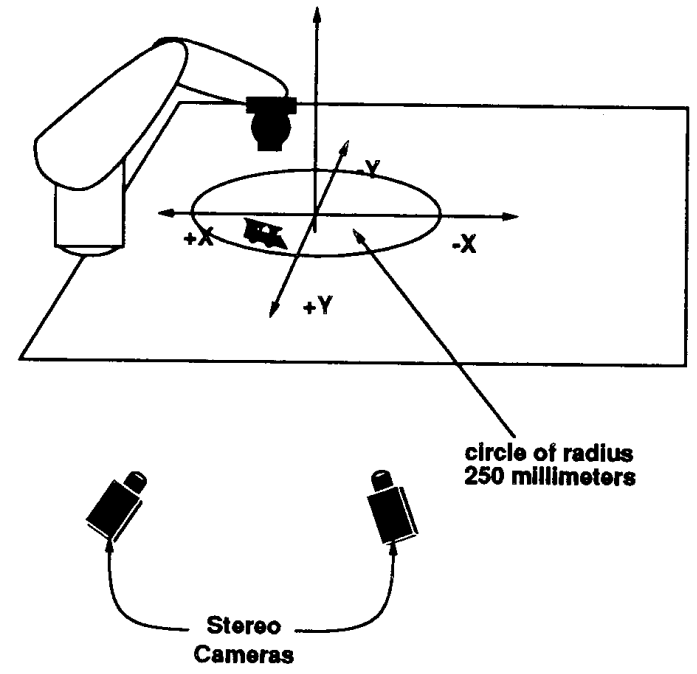

Fig. 1. Tracking/grasping system.

trajectory parameters which can be used for forward prediction, and the updated position is sent to the trajectoryplanner/arm-control system.

3) The trajectory planner updates the joint-level servos of the arm via kinematic transform equations. An additional fixed-gain filter is used to provide servo-level control in case of missed or delayed communication from the vision and filtering system.

4) Once tracking is stable, the system commands the arm to intercept the moving object and the hand is used to grasp the object stably and pick it up.

The following sections of the paper describe each of these subsystems in detail along with experimental results.

\section{PREVIOUS WORK}

Previous efforts in the areas of motion tracking and realtime control are too numerous to exhaustively list here. We instead list some notable efforts that have inspired us to use similar approaches. Burt et al. [9] have focused on highspeed feature detection and hierarchical scaling of images in order to meet the real-time demands of surveillance and other robotic applications. Related work has been reported by Lee and Wohn [29] and Wiklund and Granlund [43] who use image differencing methods to track motion. Corke, Paul, and Wohn [13] report a feature-based tracking method that uses special-purpose hardware to drive a servo controller of an arm-mounted camera. Goldenberg et al. [16] have developed a method that uses temporal filtering with vision hardware similar to our own. Luo, Mullen, and Wessel [30] report a realtime implementation of motion tracking in 1-D based on Horn and Schunk's method. Verghese et al. [41] report real-time short-range visual tracking of objects using a pipelined system similar to our own. Safadi [37] uses a tracking filter similar to our own and a pyramid-based vision system, but few results are reported with this system. Rao and Durrant-Whyte [36] have implemented a Kalman filter-based decentralized tracking

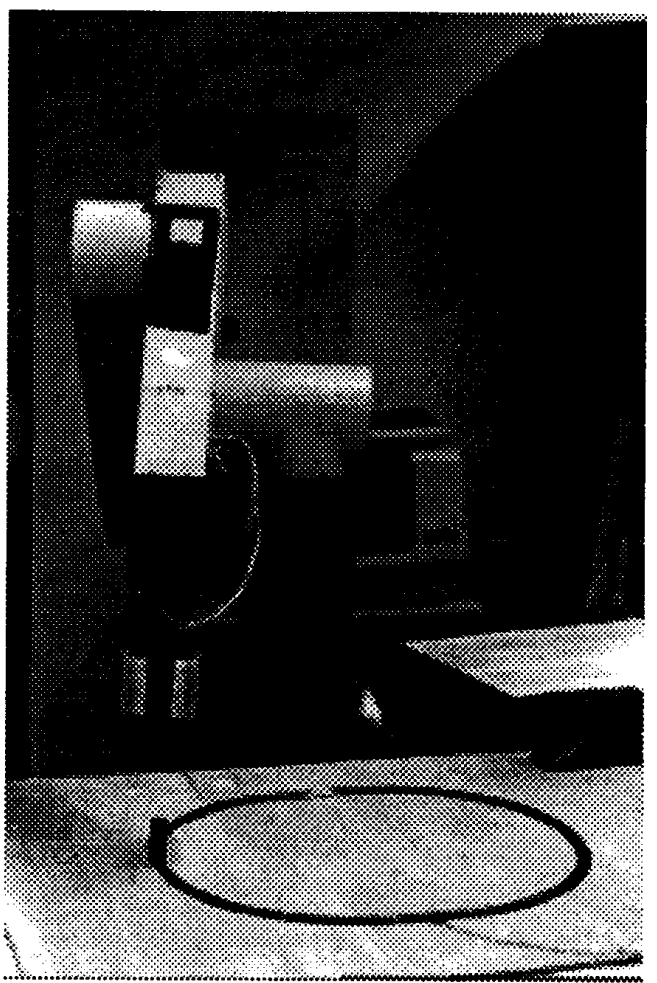

Fig. 2. Experimental hardware

system that tracks moving objects with multiple cameras. Miller [31] has integrated a camera and arm for a tracking task where the emphasis is on learning kinematic and control parameters of the system. Weiss et al. [42] also use visual feedback to develop control laws for manipulation. Brown [8] has implemented a gaze control system that links a robotic "head" containing binocular cameras with a servo controller that allows one to maintain a fixed gaze on a moving object. Clark and Ferrier [12] also have implemented a gaze control system for a mobile robot. A variation of the tracking problems is the case of moving cameras. Some of the papers addressing this interesting problem are [9], [15], [44], and [18].

The majority of literature on the control problems encountered in motion tracking experiments is concerned with the problem of generating smooth, up-to-date trajectories from noisy and delayed outputs from different vision algorithms. Our previous work [4] coped with that problem in a similar way as in [38], using an $\alpha-\beta-\gamma$ filter, which is a form of steady-state Kalman filter. Other approaches can be found in papers by [33], [34], [28], [6]. In the work of Papanikolopoulos et al. [33], [34], visual sensors are used in the feedback loop to perform adaptive robotic visual tracking. Sophisticated control schemes are described which combine a Kalman filter's estimation and filtering power with an optimal (LQG) controller which computes the robot's motion. The vision system uses an optic-flow computation based on the SSD (sum of squared differences) method which, while time consuming, appears to be accurate enough for the tracking 
task. Efficient use of windows in the image can improve the performance of this method. The authors have presented good tracking results, as well as stated that the controller is robust enough so the use of more complex (time-varying LQG) methods is not justified. Experimental results with the CMU Direct Drive Arm II show that the methods are quite accurate, robust, and promising.

The work of Lee and Kay [28] addresses the problem of uncertainty of cameras in the robot's coordinate frame. The fact that cameras have to be strictly fixed in robot's frame might be quite annoying since each time they are (most often incidentally) displaced, one has to undertake a tedious job of their recalibration. Again, the estimation of the moving object's position and orientation is done in the Cartesian space and a simple error model is assumed. Andersen et al. [6] adopt a 3rd-order Kalman filter in order to allow a robotic system (consisting of two degrees of freedom) to play the labyrinth game. A somewhat different approach has been explored in the work of Houshangi [24] and Koivo et al. [27]. In these works, the autoregressive (AR) and autogressive moving-average with exogenous input (ARMAX) models are investigated for visual tracking.

\section{VISION SYSTEM}

In a visual tracking problem, motion in the imaging system has to be translated into 3-D scene motion. Our approach is to initially compute local optic-flow fields that measure image velocity at each pixel in the image. A variety of techniques for computing optic-flow fields have been used with varying results including matching-based techniques [5], [10], [39], gradient-based techniques [23], [32], [11], and spatiotemporal energy methods [20], [2]. Optic-flow was chosen as the primitive upon which to base the tracking algorithm for the following reasons.

- The ability to track an object in three dimensions implies that there will be motion across the retinas (image planes) that are imaging the scene. By identifying this motion in each camera, we can begin to find the actual 3-D motion.

- The principal constraint in the imaging process is high computational speed to satisfy the update process for the robotic arm parameters. Hence, we needed to be able to compute image motion quickly and robustly. The Horn-Schunck optic-flow algorithm (described below) is well suited for real-time computation on our PIPE image processing engine.

- We have developed a new framework for computing optic-flow robustly using an estimation-theoretic framework [40]. While this work does not specifically use these ideas, we have future plans to try to adapt this algorithm to such a framework.

Our method begins with an implementation of the Horn-Schunck method of computing optic-flow [22]. The underlying assumption of this method is the optic-flow constraint equation, which assumes image irradiance at time $t$ and $t+\delta t$ will be the same:

$$
I(x+\delta x, y+\delta y, t+\delta t)=I(x, y, t) .
$$

If we expand this constraint via a Taylor series expansion, and drop second- and higher-order terms, we obtain the form of the constraint we need to compute normal velocity:

$$
I_{x} u+I_{y} v+I_{t}=0
$$

where $u$ and $v$ are the velocities in image space, and $I_{x}$, $I_{y}$, and $I_{t}$ are the spatial and temporal derivatives in the image. This constraint limits the velocity field in an image to lie on a straight line in velocity space. The actual velocity cannot be determined directly from this constraint due to the aperture problem, but one can recover the component of velocity normal to this constraint line as

$$
V_{n}=\frac{-I_{t}}{\sqrt{I_{x}^{2}+I_{y}^{2}}} .
$$

A second, iterative process is usually employed to propagate velocities in image neighborhoods, based upon a variety of smoothness and heuristic constraints. These added neighborhood constraints allow for recovery of the actual velocities $u$, $v$ in the image. While computationally appealing, this method of determining optic-flow has some inherent problems. First, the computation is done on a pixel-by-pixel basis, creating a large computational demand. Second, the information on optic flow is only available in areas where the gradients defined above exist.

We have overcome the first of these problems by using the PIPE image processor [26], [7]. The PIPE is a pipelined parallel image processing computer capable of processing 256 $\times 256 \times 8$ bit images at frame rate speeds, and it supports the operations necessary for optic-flow computation in a pixelparallel method (a typical image operation such as convolution, warping, addition/subtraction of images can be done in one cycle $-1 / 60 \mathrm{~s})$. The second problem is alleviated by our not needing to know the actual velocities in the image. What we need is the ability to locate and quantify gross image motion robustly. This rules out simple differencing methods which are too prone to noise and will make location of image movement difficult. Hence, a set of normal velocities at strong gradients is adequate for our task, precluding the need to iteratively propagate velocities in the image.

\section{A. Computing Normal Optic-Flow in Real-Time}

Our goal is to track a single moving object in real time. We are using two fixed cameras that image the scene and need to report motion in 3-D to a robotic arm control program. Each camera is calibrated with the 3-D scene, but there is no explicit need to use registered (i.e., scan-line coherence) cameras. Our method computes the normal component of optic-flow for each pixel in each camera image, finds a centroid of motion energy for each image, and then uses triangulation to intersect the back-projected centroids of image motion in each camera. Four processors are used in parallel on the PIPE. The processors are assigned as four per camera-two each for the calculation of $X$ and $Y$ motion energy centroids in each image. We also use a special processor board (ISMAP) to perform real-time 


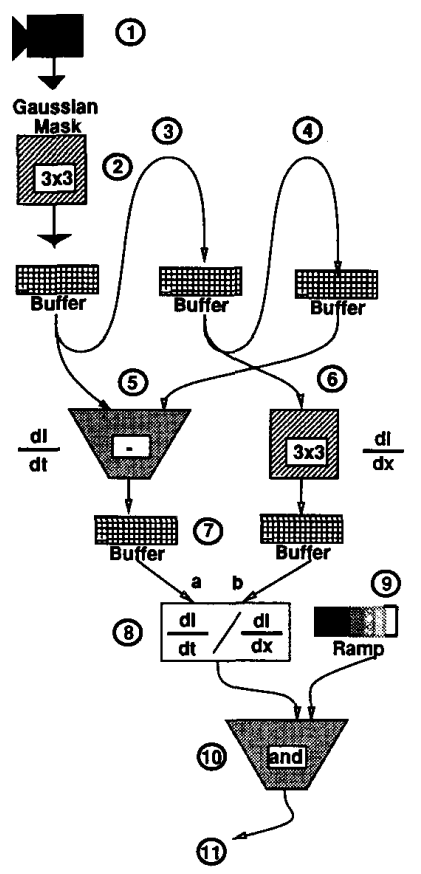

Fig. 3. PIPE motion tracking algorithm.

histogramming. The steps below correspond to the numbers in Fig. 3.

1) The camera images the scene and the image is sent to processing stages in the PIPE.

2) The image is smoothed by convolution with a Gaussian mask. The convolution operator is a built-in operation in the PIPE and it can be performed in one frame cycle.

3-4) In the next two cycles, two more images are read in, smoothed and buffered, yielding smoothed images $I_{0}$ and $I_{1}$ and $I_{2}$. The ability to buffer and pipeline images allows temporal operations on images, albeit at the cost of processing delays (lags) on output. There are now three smoothed images in the PIPE, with the oldest image lagging by $3 / 60 \mathrm{~s}$.

5) Images $I_{0}$ and $I_{2}$ are subtracted yielding the temporal derivative $I_{t}$.

6) In parallel with step 5, image $I_{1}$ is convolved with a $3 \times 3$ horizontal spatial gradient operator, returning the discrete form of $I_{x}$. In parallel, the vertical spatial gradient is calculated yielding $I_{y}$ (not shown).

7-8) The results from steps 5 and 6 are held in buffers and then are input to a look-up table that divides the temporal gradient at each pixel by the absolute value of the summed horizontal and vertical spatial gradients [which approximates the denominator in (3)]. This yields the normal velocity in the image at each pixel. These velocities are then thresholded and any isolated (i.e., single pixel motion energy) blobs are morphologically eroded. The above threshold velocities are then encoded as gray value 255 . In our experiments, we thresholded all velocities below 10 pixels per $60 \mathrm{~ms}$ to zero velocity.
9-10) In order to get the centroid of the motion information, we need the $X$ and $Y$ coordinates of the motion energy. For simplicity, we show only the situation for the $X$ coordinate. The gray-value ramp in Fig. 3 is an image that encodes the horizontal coordinate value $(0-255)$ for each point in the image as a gray value. Thus, it is an image that is black (0) at horizontal pixel 0 and white (255) at horizontal pixel 255 . If we logically and each pixel of the above threshold velocity image with the ramp image, we have an image which encodes high velocity pixels with their positional coordinates in the image, and leaves pixels with no motion at zero.

11) By taking this result and histogramming it, via a special stage of the PIPE which performs histograms at frame rate speeds, we can find the centroid of the moving object by finding the mean of the resulting histogram. Histogramming the high-velocity position encoded images yields 256 16-bit values (a result for each intensity in the image). These 256 values can be read off the PIPE via a parallel interface in about $10 \mathrm{~ms}$. This operation is performed in parallel to find the moving object's $Y$ centroid (and in paraliel for $X$ and $Y$ centroids for camera 2). The total associated delay time for finding the centroid of a moving object becomes 15 cycles or $0.25 \mathrm{~s}$.

The same algorithm is run in parallel on the PIPE for the second camera. Once the motion centroids are known for each camera, they are back-projected into the scene using the camera calibration matrices and triangulated to find the actual 3-D location of the movement. Because of the pipelined nature of the PIPE, a new $X$ or $Y$ coordinate is produced every $1 / 60$ $\mathrm{s}$ with this delay. Fig. 4 shows two camera images of a moving train, and Fig. 5 shows the motion energy derived from the real-time optic-flow algorithm.

While we are able to derive 3-D position from motionstereo at real-time rates, there are a number of sources of noise and error inherent in the vision system. These include stereotriangulation error, moving shadows which are interpreted as object motion (we use no special lighting in the scene), and small shifts in centroid alignments due to the different viewing angles of the cameras, which have a large baseline. The net effect of this is to create a 3-D position signal that is accurate enough for gross-level object tracking, but is not sufficient for the smooth and highly accurate tracking required for grasping the object. We describe in the next section how a probabilistic model of the motion that includes noise can be used to extract a more stable and accurate 3-D position signal.

\section{ROBOTIC ARM CONTROL}

The second part of the system is the arm control. The robotic arm has to be controlled in real time to follow the motion of the object, using the output of the vision system. The raw vision system output is not sufficient as a control parameter since its output is both noisy and delayed in time. The control system needs to do the following: 


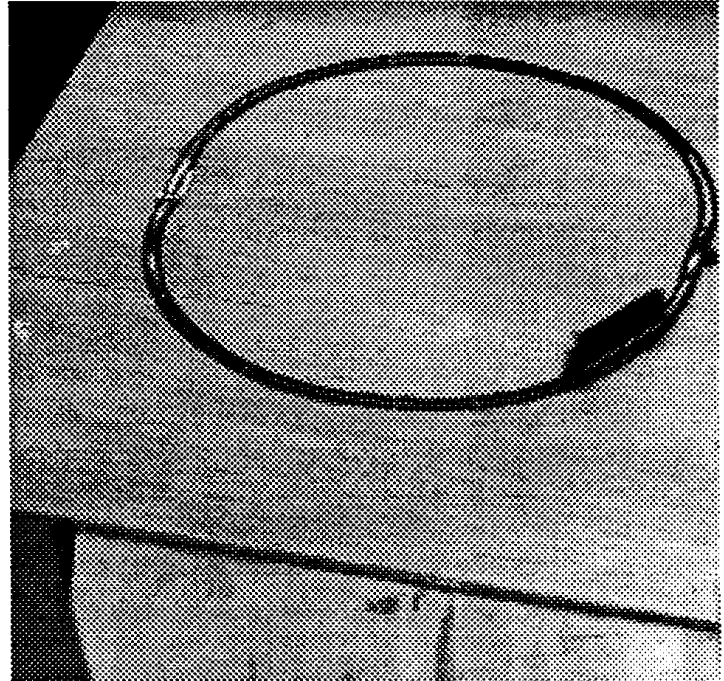

(a)

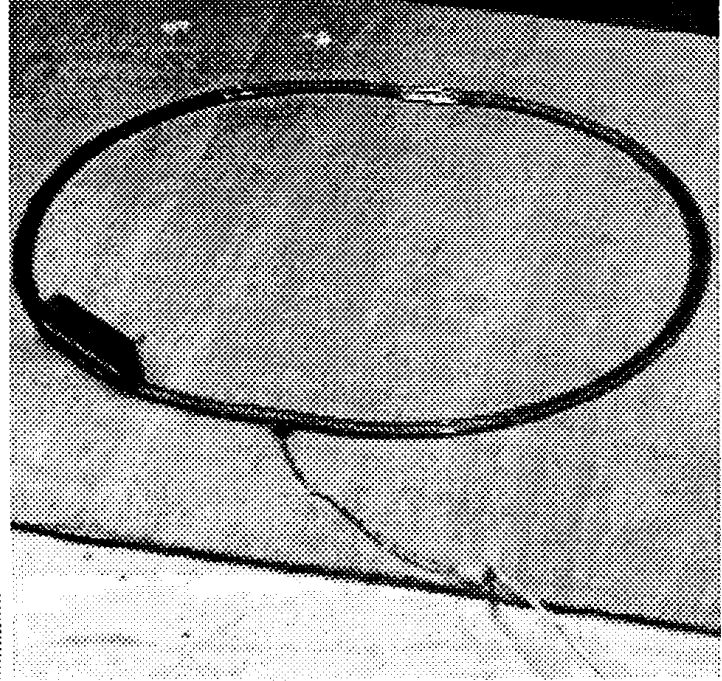

(b)

Fig. 4. Left and right camera images

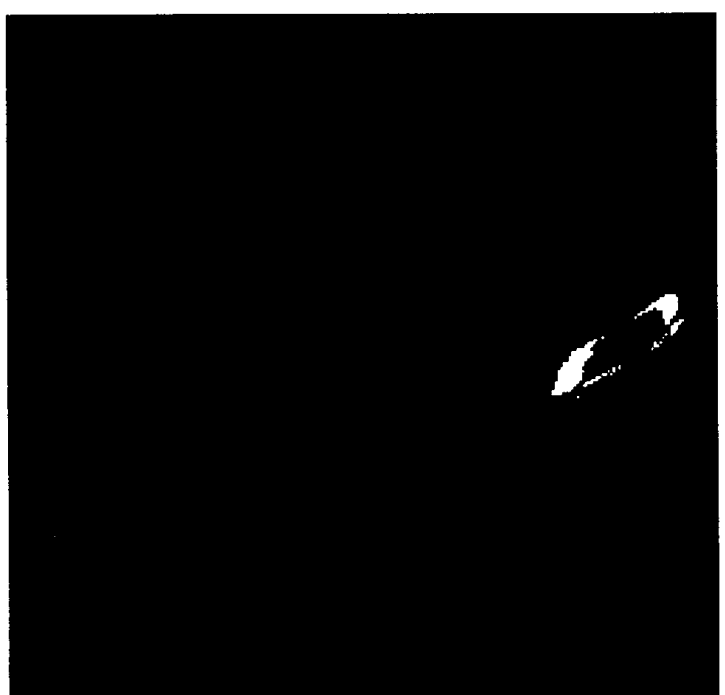

(a)

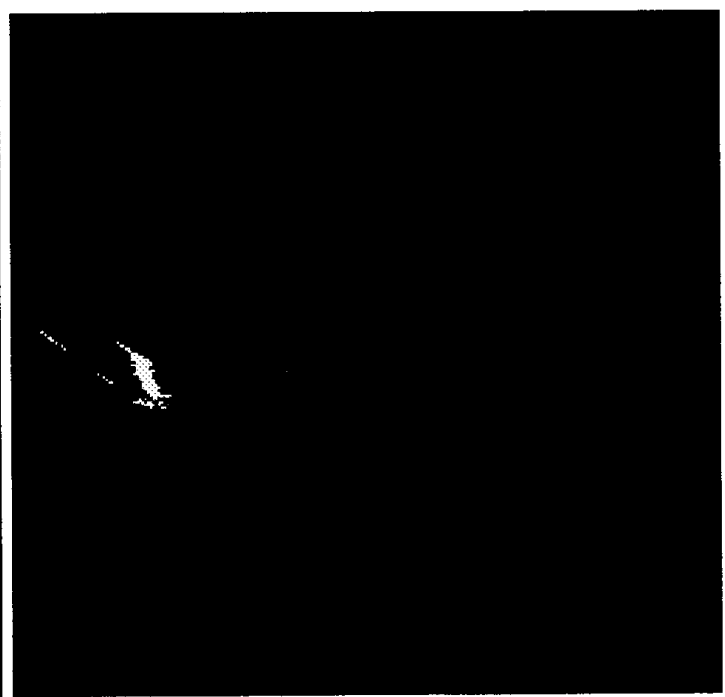

(b)

Fig. 5. Motion energy derived from optic flow (left and right cameras)

- filter out the noise with a digital filter:

- predict the position to cope with delays introduced by both vision subsystem and the digital filter;

- perform the kinematic transformations which will map the desired manipulator's tip position from a Cartesian coordinate frame into joint coordinates, and actually perform the movement.

Our vision algorithm provides in each sampling instant a position in space as a triplet of Cartesian coordinates $(x, y, z)$. The task of the control algorithm is to smooth and predict the trajectory, thus positioning the robot where the object is during its motion.
A well-known and useful solution is the Kalman filter approach, because it successfully performs both smoothing and prediction. However, the assumption the Kalman filter makes is that the noise applied to the system is white. That fact directly depends on the parametrization of the trajectory and, unfortunately in our case, the simplest possible parametrization - Cartesian--does not support this noise model. Our previous work [4] used a variant of this approach and obtained tracking that was smooth but not accurate enough to allow actual grasping of the moving object. Our solution to this problem was to appeal to a local coordinate system that was able to model the motion and system noise characteristics 


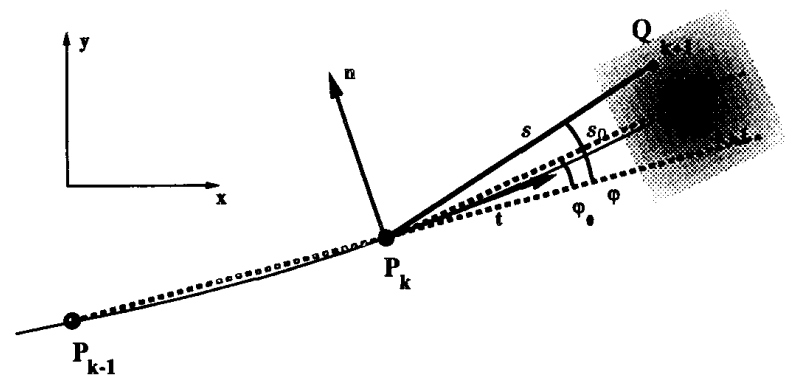

Fig. 6. Model of the motion in the plane: the moving object is in $P_{k+1}$, while the vision system computes $Q_{k+1} . s_{0}$ is the actual arc length, and $s$ is the measured arc length.

more accurately, thus producing a more accurate control algorithm.

\section{A. The Model of the Motion}

The model of the motion we are using separates 3-D space into an $X Y$ plane and the $Z$ axis, and addresses these two components of motion separately. In the experimental results we present in Section VI, we have used the model to track planar trajectories. However, we still need to track the $Z$ dimension to determine the height above the plane of the motion to command the robotic arm to correctly grasp the object. The tracking of the object in the $X Y$ plane is done using a local model presented below, while the tracking in $Z$ is done with a Cartesian displacement using the fixed-gain filter described in [4].

The main idea in the trajectory parametrization used in this paper is to describe a point in a local coordinate frame, relative to the point from the previous sampling instant, by the triplet of coordinates $\left(s_{0}, \phi_{0}, \Delta z\right)$ where (see Fig. 6)

- $s_{0}$ is the length of an arc between two points (we will approximate the arc length by a straight line section and set $s_{0}$ to be the distance between points $P_{k}$ and $P_{k+1}$, $s_{0}=\left\|P_{k+1}-P_{k}\right\|$ where $\|\cdot\|$ denotes Euclidean distance);

- $\phi_{0}$ is the "bending" of the trajectory;

- $\Delta z$ is the altitude difference between two consecutive points.

Due to the existence of noise, the measured coordinates will be random variables with certain distributions. We have made the following assumptions, as a result of both reasoning about the vision algorithm and certain necessary simplifications.

- In sampling instant $k$, our object is at point $P_{k}$.

- In the next sampling instant $k+1$, the object is at $P_{k+1}$ and the point returned by the vision algorithm is $Q_{k+1}$. The measured arc length is $s=\left\|Q_{k+1}-P_{k}\right\|$.

- $Q_{k+1}$ is normally distributed around $P_{k+1}$. The noise can be expressed by its two components, tangential $n_{t}$ and normal $n_{n}$, where both $n_{t}$ and $n_{n}$ are random variables.

- $n_{t}$ and $n_{n}$ are both zero-mean, with the same variance $\sigma$ and mutually not correlated. Experimentally, it has been determined that their coefficient of correlation is between 0.1 and 0.2 .

- $\Delta z$ is independent of $s_{0}$ and $\phi_{0}$ (i.e., the altitude $Z$ is independent of the position in $X Y$ plane).

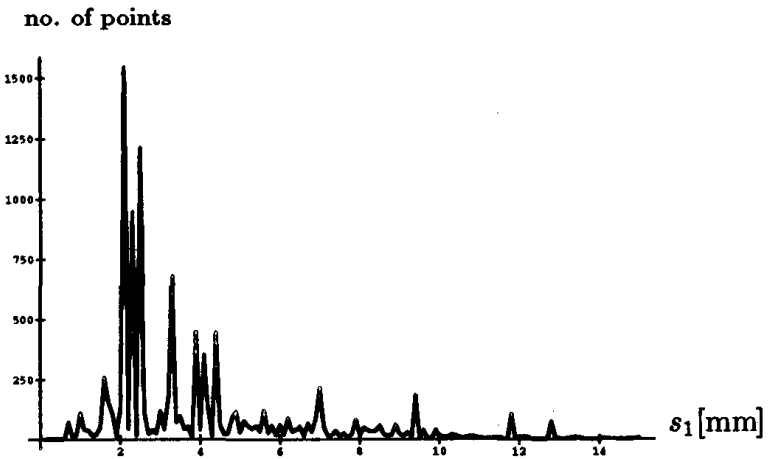

Fig. 7. Experimental density of $s_{1}$, the expected value of the arc length.

This last assumption limits the method to tracking planar trajectories in space. However, the probabilistic method described below can be extended to arbitrary 3-D space curve trajectories by finding a distribution that will allow computation of a space curve torsion parameter. This essentially means creating a full Frenet Frame representation at each point in time.

Under these assumptions, it can be shown that (see Appendix I) the velocity $v$ and curvature $\kappa$ are

$$
\begin{aligned}
& v=\lim _{T \rightarrow 0} s_{0} / T \\
& \kappa=\lim _{T \rightarrow 0} \tan \phi_{0} / s_{0}
\end{aligned}
$$

where $s_{0}=\left\|P_{k+1}-P_{k}\right\|, \phi_{0}=\pi-\angle P_{k-1} P_{k} P_{k+1}$ and $T$ is the sampling interval.

Our model assumes the following coordinate transformation that relates the moving object's coordinate frame at one instant with the next instant in time:

$$
T_{\Delta}=\operatorname{rot}\left(z, \phi_{0}\right) \circ \operatorname{trans}\left(x, s_{0}\right) \circ \operatorname{trans}(z, \Delta z)
$$

where rot and trans are rotation about and translation along a given axis. Presented as a $4 \times 4$ matrix, transformation (6) is

$$
T_{\Delta}=\left[\begin{array}{cccc}
\cos \phi_{0} & -\sin \phi_{0} & 0 & s_{0} \cos \phi_{0} \\
\sin \phi_{0} & \cos \phi_{0} & 0 & s_{0} \sin \phi_{0} \\
0 & 0 & 1 & \Delta z \\
0 & 0 & 0 & 1
\end{array}\right] .
$$

What are the advantages of such a parametrization? The most obvious one is the simplicity of the prediction task in this framework; all we need is to multiply the velocity $v=s_{0} / T$ by the time $\tau>T$ we want to predict, as well as "bending" $\phi_{0}$. The next advantage is that in order to achieve an accurate prediction, we do not need a high-order model with the mostly heuristic tuning of numerous parameters. The price we have to pay is that filtering is not straightforward. It turns out that we cannot just apply a low-pass filter in order to recover a dc component from $s$, but rather we need a more elaborate approach that takes into account a probabilistic distribution of $s$. Fig. 7 is a histogram of the experimentally measured density of the computed arc length between triangulated image motion points. This distribution shows the need to use a more sophisticated method than a simple averaging filter, which we have found to be incorrect in being able to correctly estimate the movement of the object between vision samples. 


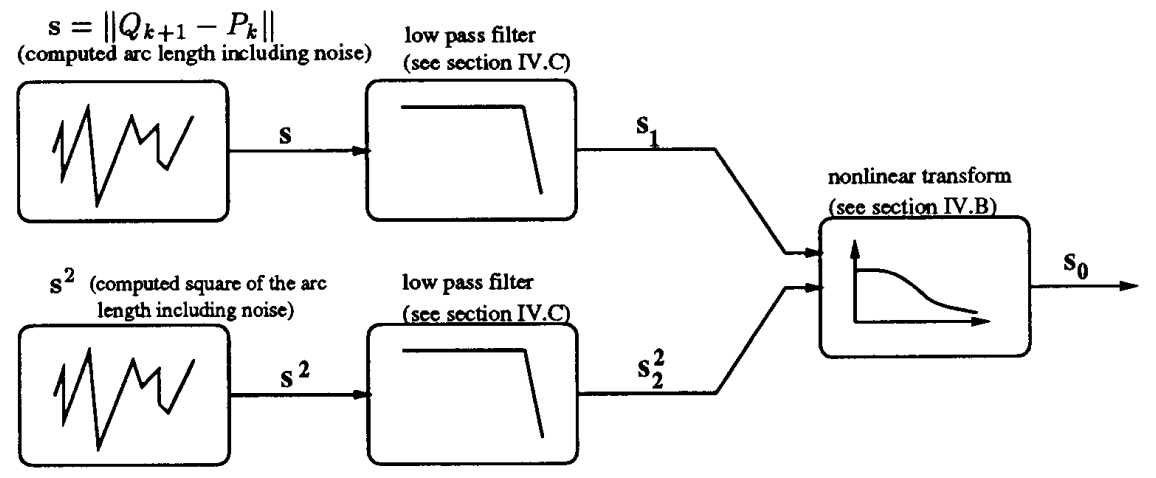

Fig. 8. Overview of the filtering method.

The analysis below describes a probabilistic model of the experimental distribution in Fig. 7, allowing us to recover the actual arc length parameter $s_{0}$ and the bending angle $\phi_{0}$ at each sampling instant. While this model introduces more complexity than a standard Cartesian model, we will see below that it is more effective in allowing us to accurately predict and smooth our trajectory.

\section{B. Estimating Arc Length $s_{0}$ and Bending Parameter $\phi_{0}$}

We begin this subsection with an intuitive overview of the method used to recover the actual arc length $s_{0}$ and actual bending parameter $\phi_{0}$ from the noisy estimates provided by the vision system. The filtering method is summarized in Fig. 8. Given the model of the motion described in the previous subsection, we want to extract the actual arc length $s_{0}$ from measured, noisy arc length $s$. Using a smoothing filter (see subsection $\mathrm{C}$ ), we can derive the expected value of the arc length which we denote $s_{1}$, as well as its second-order moment (expectation of $s^{2}$ ) which we denote $s_{2}^{2}$. These two control inputs, $s_{1}$ and $s_{2}^{2}$, can be used to create two integral equations [(13) and (15)] which, when integrated, express the known smoothed control inputs $s_{1}$ and $s_{2}^{2}$ as functions of the actual arc length $s_{0}$ and a variance $\sigma$. These equations allow us to estimate $s_{0}$ from the control inputs. To recover the actual bending parameter $\phi_{0}$, our task is simplified since its distribution is symmetric, and its expectation is the actual bending parameter itself.

Let $s=\left\|Q_{k+1}-P_{k}\right\|$ be the distance between the object and the next position returned by the vision algorithm. Let $\mathcal{F}_{k}$ be the moving coordinate frame of the point $P_{k}$ with axes $\boldsymbol{t}, \boldsymbol{n}$, and $Z$, where $\boldsymbol{t}$ is the tangential and $\boldsymbol{n}$ normal to the trajectory's projection in $X Y$ plane (see Fig. 6), and let $\mathcal{F}_{k+1}$ be the analogous coordinate frame in the point $P_{k+1}$. The transformation from $\mathcal{F}_{k}$ to $\mathcal{F}_{k+1}$ is given by $T_{\Delta}$ :

$$
\mathcal{F}_{k+1}=T_{\Delta} \mathcal{F}_{k}
$$

The point $Q_{k+1}$ is given by $\mathcal{F}_{k+1}$ by a triple $\left(n_{t}, n_{n}, z\right)$, where $n_{t}$ is a noise component along $t$, and $n_{n}$ is a noise component along $\boldsymbol{n}$. Both $n_{t}$ and $n_{n}$ are Gaussian with zero mean and mutually noncorrelated. Coordinate $z$ is the altitude at $P_{k+1}$.
In the coordinate frame $\mathcal{F}_{k}$, the point $Q_{k+1}$ is given by $T_{\Delta}^{-1}\left[\begin{array}{llll}n_{t} & n_{n} & z & 1\end{array}\right]^{T}$. Thus, the distance $s=\left\|Q_{k+1}-P_{k}\right\|$ is given by

$$
\begin{aligned}
s & =\left\|\left[\begin{array}{cc}
\cos \phi_{0} & \sin \phi_{0} \\
-\sin \phi_{0} & \cos \phi_{0}
\end{array}\right]\left[\begin{array}{c}
n_{t} \\
n_{n}
\end{array}\right]+\left[\begin{array}{c}
s_{0} \\
0
\end{array}\right]\right\| \\
& =\sqrt{\left(n_{t}^{\prime}+s_{0}\right)^{2}+n_{n}^{\prime 2}}
\end{aligned}
$$

where $n_{t}^{\prime}=n_{t} \cos \phi_{0}+n_{n} \sin \phi_{0}$ and $n_{n}^{\prime}=-n_{t} \sin \phi_{0}+$ $n_{n} \cos \phi_{0} . n_{t}^{\prime}$ and $n_{n}^{\prime}$ are obtained by "rotating" $n_{t}$ and $n_{n}$ by $\phi_{0}$. It is known that the transformation of nolicorrelated Gaussian random variables results in noncorrelated Gaussian random variables with the same variance. Thus, $n_{t}^{\prime}$ and $n_{n}^{\prime}$ are noncorrelated and Gaussian with the variance $\sigma$.

Now we have expressed in relation (8) the dependency of $s$ on two random variables with known properties $n_{t}^{\prime}$ and $n_{n}^{\prime}$. The formula for random variables' distribution transformations gives us the distribution function $F(s)$. (Note that henceforth $s$ is used to denote the argument of the distribution or distribution density functions.)

$$
F(s)=\iint_{D} \frac{1}{2 \pi \sigma^{2}} \mathrm{e}^{-\frac{1}{2}\left[\left(\frac{t-s_{0}}{\sigma}\right)^{2}+\left(\frac{n}{\sigma}\right)^{2}\right]} \mathrm{d} t \mathrm{~d} n
$$

where $D$ is a disk of radius $s$ about $P_{k}$.

We need to find the expectation of the random variable whose distribution is given by (9). In order to do that, we need the density function which can be found by calculating the integral in (9). By introducing the substitution $t=$ $r \cos \theta, n=r \sin \theta$, we get

$$
F(s)=\frac{1}{2 \pi \sigma^{2}} \int_{0}^{s} r \int_{0}^{2 \pi} \mathrm{e}^{-\frac{1}{2}\left[\left(\frac{r \cos \theta-s_{0}}{\sigma}\right)^{2}+\left(\frac{r \sin \theta}{\sigma}\right)^{2}\right]} \mathrm{d} \theta \mathrm{d} r .
$$

The distribution density is given as $f(s)=\frac{\mathrm{d} F(s)}{\mathrm{d} s}$ or, after differentiation,

$$
f(s)=\frac{s \mathrm{e}^{-\frac{s^{2}+s_{0}^{2}}{2 \sigma^{2}}}}{2 \pi \sigma^{2}} \int_{0}^{2 \pi} \mathrm{e}^{-\frac{s s_{0}}{\sigma^{2}} \cos \theta} \mathrm{d} \theta .
$$

The last integral can be expressed by a modified Bessel function $I_{0}(z)$

$$
f(s)=\frac{s}{\sigma^{2}} \mathrm{e}^{-\frac{s^{2}+s_{0}^{2}}{2 \sigma^{2}}} I_{0}\left(\frac{s s_{0}}{\sigma^{2}}\right) .
$$




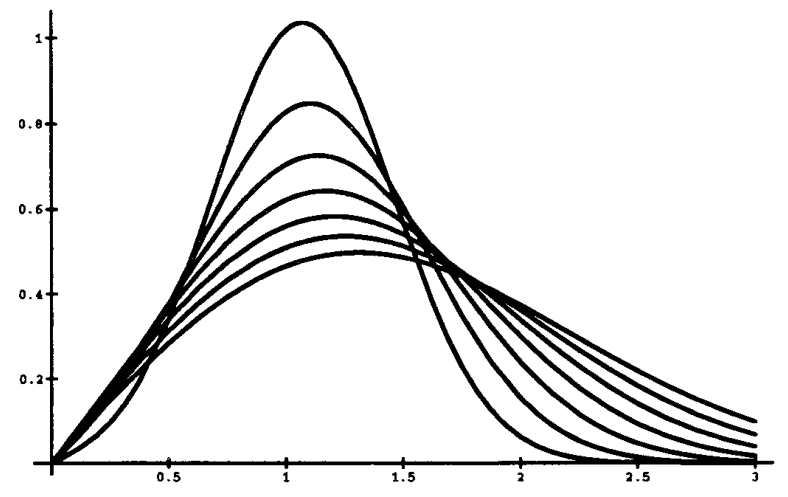

Fig. 9. Distribution density $f(s), s_{0}=1, \sigma=0.4-1.0$, increment $=0.1$.

A graph of $f(s)$ is given in Fig. 9. Here, $s_{0}$ is fixed to 1 , and $\sigma$ varies from 0.4 to 1.0 . Our job is to recover $s_{0}$ given $f(s)$.

It is apparent from Fig. 9 that the peak value of $f(s)$ depends on $\sigma$, and drifts toward higher values as $\sigma$ grows. The expectation for $s$ also depends on $\sigma$. In particular, we have

$$
s_{1}=E(s)=\int_{0}^{\infty} s f(s) \mathrm{d} s=\sigma u\left(\frac{s_{0}}{\sigma}\right)
$$

where

$$
\begin{aligned}
u(x)= & \sqrt{\frac{\pi}{2}} \mathrm{e}^{-x^{2} / 4} \\
& \cdot\left(I_{0}\left(\frac{x^{2}}{4}\right)+\frac{x^{2}}{2}\left(I_{0}\left(\frac{x^{2}}{4}\right)+I_{1}\left(\frac{x^{2}}{4}\right)\right)\right) .
\end{aligned}
$$

Here, $\sigma$ is the constant for the given system and it is related to $s_{0}$. In order to estimate $\sigma$, we will use the second-order moment

$$
s_{2}^{2}=E\left(s^{2}\right)=\int_{0}^{\infty} s^{2} f(s) \mathrm{d} s=s_{0}^{2}+2 \sigma^{2} .
$$

Equations (13) and (15) are derived in Appendix II.

Now by eliminating $s_{0}$ from (13) and (15), we have

$$
1=z u\left(\frac{\sqrt{p^{2}-2 z^{2}}}{z}\right)
$$

where $p=s_{2} / s_{1}$ and $z=\sigma / s_{1}$. Now by setting $x=$ $\sqrt{\frac{p^{2}-2 z^{2}}{z}}$, we end up with an equation (see Appendix III)

$$
u_{1}(x)=\frac{\sqrt{x^{2}+2}}{u(x)}=p .
$$

Equation (17) relates our known control inputs $\left(p=s_{2} / s_{1}\right)$ to $x$. We can create a table of values for this function off line, and then by interpolation calculate a value of $x$ given $p$.

Let $x_{0}(p)$ be the solution of (17). Now we can express $s_{0}$ and $\sigma$ as functions of $s_{1}$ and $s_{2}$ as follows:

$$
s_{0}=s_{2} \frac{x_{0}\left(\frac{s_{2}}{s_{1}}\right)}{\sqrt{2+x_{0}\left(\frac{s_{2}}{s_{1}}\right)^{2}}}
$$

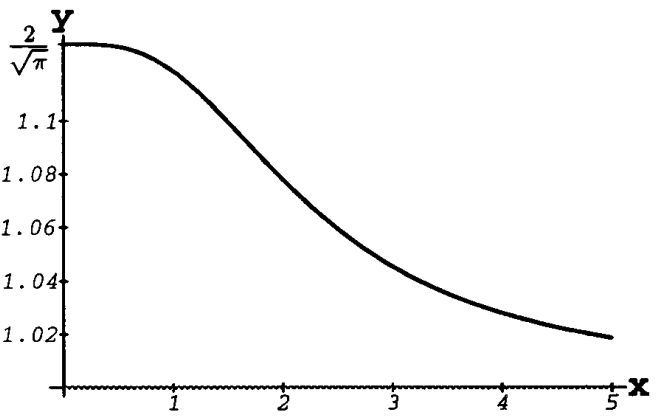

Fig. 10. $y=u_{1}(x)$.

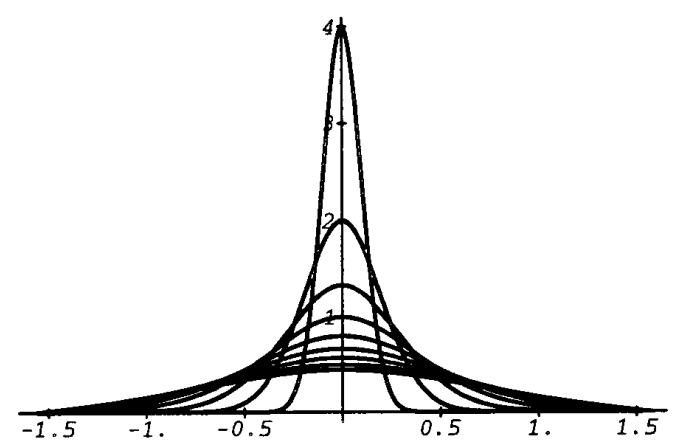

Fig. 11. Distribution density $f(\phi)$.

$$
\sigma=s_{2} \frac{1}{\sqrt{2+x_{0}\left(\frac{s_{2}}{s_{1}}\right)^{2}}}
$$

This method requires little on-line computation-an interpolation table of values of $u_{1}$ is all we need to recover the arc length parameter $s_{0}$.

To find the bending parameter $\phi_{0}$, we use the same technique as for the distribution of $s$, and we get the following formula:

$$
f(\phi)=\frac{\cos \left(\phi-\phi_{0}\right)}{\sqrt{2 \pi} k} \mathrm{e}^{-\frac{\sin ^{2}\left(\phi-\phi_{0}\right)}{2 k^{2}}}
$$

where $k=\sigma / s_{0}$ and $\phi-\phi_{0} \in(-\pi / 2, \pi / 2)$. It is obvious that $f$ is symmetric around $\phi_{0}$, which also means that the expectation $E \phi=\phi_{0}$. Hence, we so not need to perform a nonlinear filtering to recover $\phi_{0}$.

The graph of $f$ for $k=0.1$ to 0.9 and $\phi_{0}=0$ is given in Fig. 11.

\section{Smoothing of the Control Inputs}

In the previous subsection, we showed how to extract parameters $s_{0}$ and $\phi_{0}$ from the updated positions determined from the vision system. The signals $s_{1}, s_{2}^{2}$ described in (13) and (15) are in fact the smoothed versions of the expectations of the control signals $s, s^{2}$ which are the arc length and the arc length squared. The smoothing filter we use to compute these signals is a moving-average (MA) filter using a Kaiser window [25]. This filter provides the largest ratio of signal 


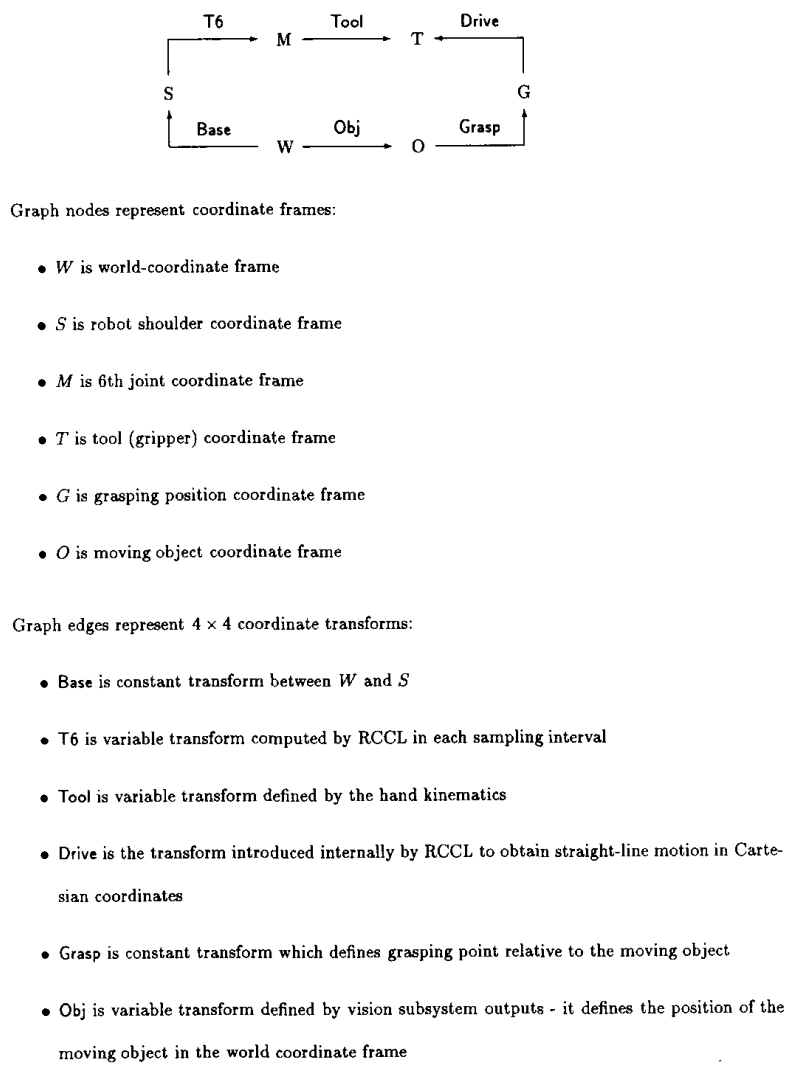

Fig. 12. Transform equation.

energy in the main lobe and a side lobe, which usually results in a filter of lower order. The windowing function is given by

$$
w_{K}(n)=\frac{I_{0}\left(\beta \sqrt{\left.1-(1-2 n / M)^{2}\right)}\right.}{I_{0}(\beta)}
$$

where $I_{0}$ is the modified zeroth-order Bessel function, $\beta$ is the shape parameter which defines the width of the main lobe, and $M$ is the order of the filter. According to [25], $\beta$ and $M$ are given by

$$
M \approx \frac{A-7.95}{14.36 \Delta \omega}
$$

and

$\beta= \begin{cases}0.1102(A-8.7), & A \geq 50 \\ 0.5842(A-21)^{0.4}+0.07886(A-21), & 21<A<50\end{cases}$

where $A$ is the stopband attenuation and $\Delta \omega=\left(\omega_{r}-\right.$ $\left.\omega_{c}\right) / \omega_{s}, \omega_{r}$ is the stopband frequency, $\omega_{c}$ is the passband frequency, and $\omega_{s}$ is the sampling frequency.

We have adopted $A=30$ and $\Delta \omega=0.05$ which results in $M=30$. Since the frequency of the vision algorithm is about $60 \mathrm{~Hz}$, the overall length of the window is about $0.5 \mathrm{~s}$. We also apply this MA filter to the bending parameter $\phi$.

The implementation of MA filter is straightforward: once the weights are computed off line, a window of length $M$ of measurements is retained and each sample is multiplied by an appropriate weight in the sampling period, which requires $M$ multiplications and $M-1$ additions. This allows reasonably wide windows (even up to several hundred entries) to be used in computing the smoothed signal.

\section{Prediction and Synchronization}

The host computer controls the initial vision processing and subsequent computation of control parameters described above. The host computer is able to predict the trajectory using the derivation of velocity and curvature in (4) and (5). These updated predictions are sent to the trajectory generator that is actually controlling the robot arm. The trajectory generator is a separate system that has two parallel tasks: a lowpriority task which reads the serial line receiving updated control signals, and a high-priority task which calculates the transformation equation and moves the manipulator. Those two tasks communicate via shared memory. The job of the robot controlling program is to synchronize its two tasks (i.e., to obtain mutual exclusion in accessing shared data), to unpack input packets read from the serial line, and to update the joint servos every $30 \mathrm{~ms}$.

The asynchronous nature of the communication between the host computer and the trajectory generator can result in missed or delayed communications between the two systems. Since the updating of the robotic arm parameters needs to be done at very tightly specified servo rates $(30 \mathrm{~ms})$, it is imperative that the trajectory generator can provide updated control parameters at these rates, regardless of whether it has received a new control input from the host. Therefore, we have implemented a fixed-gain $\alpha-\beta-\gamma$ filter as part of the trajectory generator [38]. This filter provides a small amount of prediction to the trajectory parameters if the control signals from the host are delayed.

We are using RCCL [19] to control the robotic arm (a PUMA 560). RCCL (Robot Control C Language) allows the use of $\mathrm{C}$ programming constructs to control the robot as well as defining transformation equations (as described in [35]). The transformation equations permit dynamic updating of arm position by generating the $4 \times 4$ transform of the moving object's position from the vision system and sending this information to the arm control algorithm (see Fig. 12).

\section{MOTOR COORDINATION FOR GRASPING}

The remaining part of our system is the interception and grasping of the object. This is the least well-developed aspect of our system, since it is a difficult real-time problem. Currently, we are intercepting and grasping a simple object with no knowledge of its local geometry. In the future, we hope to use visual information as well finger contact information to perform grasping of objects of different shapes.

We have examined the human psychological literature in order to find useful paradigms for robotic visual-motor coordination strategies that include arm movement and grasping from visual inputs. Schmidt [17] has proposed a theory of generalized motor programs, or movement schemas. In this view, a skilled action is composed of an ordered set of parametrized motor control programs of short duration (less than $200 \mathrm{~ms}$ ), each of which accomplishes one part of the task. As one program is completed, the next one is executed. At the 
initiation of a skilled task, the parameters of the motor control program are determined by sensory input and task demands, and then the programs are executed to completion. If the wrong program is selected for some reason, the program cannot be stopped by use of sensory information. An example of this can be seen in the motor activity associated with playing table tennis. In moving the arm to hit the ball, the motion of the racket is determined before the beginning of the swing and visual input has little effect after the initiation of motion. As an example of Schmidt's theory, the skilled task of grasping a moving object could be partitioned into two motor control schemas: one to position the arm and a second one to control the grasping action.

The initial strategy we have adopted in picking up the object is an open-loop strategy, similar in spirit to the preprogrammed motor control schemas described above. Schmidt's schema theory holds that for tasks of short duration, perception is used to find a set of parameters to pass to a motor control program. It is not used during the execution of a task. When grasping a moving object, for example, once vision has determined the trajectory of the object, the reach and grasping motor schemas take over with no interference from vision. Recent work described in [21] suggests that visual input may be used to modify human arm grasping trajectories, but only after a $100 \mathrm{~ms}$ or more delay after receiving the visual input. Since our visual inputs are already delayed by this amount, it may not be possible to use our current vision system effectively in this part of the task, motivating the use of open-loop schemas.

In our implementation of this strategy, vision is not used to continually monitor the grasping, but only to provide a final position and velocity from which the arm is directed to very quickly move to the object. This automatic movement is done by establishing coordinate frames of action for each of the components of the system and solving transformation equations (see Fig. 12).

The transformation equations permit dynamic updating of the arm position by generating the $4 \times 4$ transform of the moving object's position from the vision system and sending this information to the arm control algorithm. This positional information from the vision system is used to update the Obj transform in Fig. 12. The other transforms in the equation are known, and this allows the system to solve for the Drive transform which is the transform used to update the manipulator's joints and develop a straight line path in Cartesian coordinates that will bring the hand into contact with the moving object. Because the movement of the hand requires a small amount of time during which the object may have moved, the object's trajectory is predicted ahead during the movement using the $\alpha-\beta-\gamma$ predictor. By keeping the fingers of the hand spread during this maneuver, no actual contact takes place until the gripper reaches the position of the moving object. Once this position is achieved, the gripper is commanded to close and grasp the object.

\section{EXPERIMENTAL RESULTS}

We have implemented the system described above in order to demonstrate the capability of the methods. The goal was

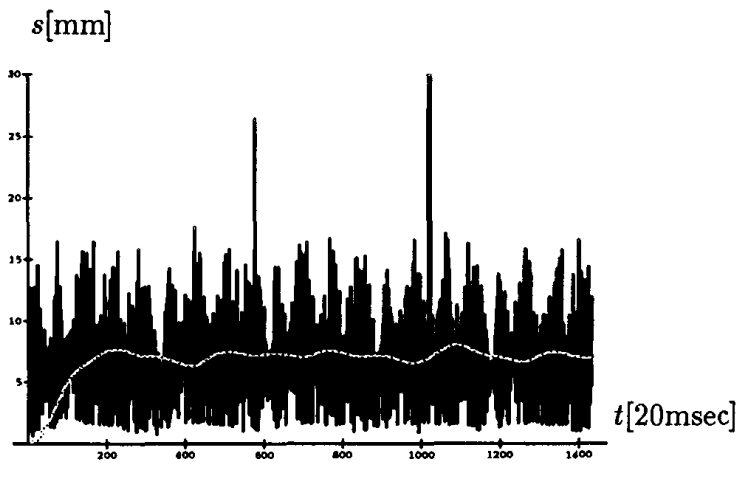

Fig. 13. Input signal $s_{1}$ (black) and filtered signal $s_{0}$ (gray). Horizontal axis is time expressed in sampling periods ( 1 unit $=20 \mathrm{~ms}$ ) and vertical axis is arc length in millimeters.

to track a moving model train, intercept it, stably grasp it, and pick it up. The train was moving in an oval trajectory; however, the system had no a priori knowledge of this particular trajectory. The velocity of the train was varied from $10-30 \mathrm{~cm} / \mathrm{s}$. We have experimentally established $100 \mathrm{~ms}$ to be the appropriate time to predict. Since the trajectory generation runs at $20 \mathrm{~ms}$, that makes five sampling intervals. The accuracy of tracking was critical; in order not to knock the train off but rather to pick it up the displacement of the robot's gripper from the actual train's position has to be in subcentimeter range.

In this section we present some results obtained by experiments. First, in Fig. 13, we have the actual measured arc length signal $s_{1}$ (black) and the filtered signal $s_{0}$ (gray). It is noticeable that $s_{0}$ is somewhat below the expected value of $s_{1}$. The nature of $s_{1}$ is quite noisy; however, the analysis described in Section IV was able to accurately extract the correct control signal. The arm control is particularly smooth and jerk free, stable over time (the tracking is continuous for many revolutions of the train) and is highly accurate in being able to intercept and grasp the object between the jaws of the gripper as it moves. Fig. 17 shows the trajectory of commanded arm control set points in the $x y$ plane, including the initial trajectory at system start. The actual train track is an oval, slightly tilted from the horizontal axis. As a part of a system, we have implemented a simple on-line graphic interface in $X$-windows environment. Fig. 18 shows the graphics window captured during an experiment. Positions are taken each 500 $\mathrm{ms}$ and plotted in the window. Figs. 14, 15, and 16 show the time dependency of the $x, y$, and $z$ coordinates. The tail of Fig. 16 is part of the grasping trajectory.

Because we are using a parallel jaw gripper, the jaws must remain aligned with the tangent to the actual trajectory of the moving object. This tangential direction is computed directly from the calculation of the bending parameter $\phi$ during the trajectory modeling phase and is used to align joint 6 of the robot to keep the gripper correctly aligned. This correct alignment allows grasping to occur at any point in the trajectory.

Fig. 19 shows three frames taken from a video tape of the system intercepting, grasping, and picking up the object. The system is quite repeatable, and is able to track other arbitrary 
$x[\mathrm{~mm}]$

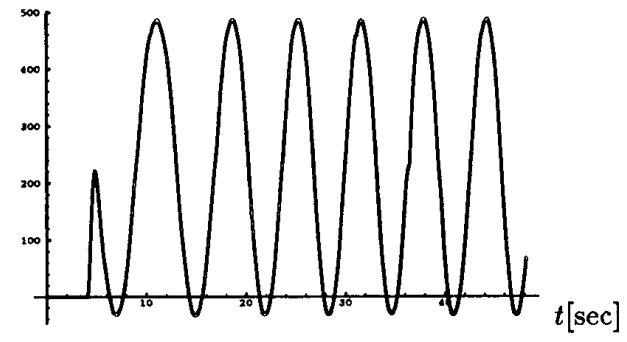

Fig. 14. $X$ coordinate of the commanded arm control set points for the tracked trajectory as a function of time.

$y[\mathrm{~mm}]$

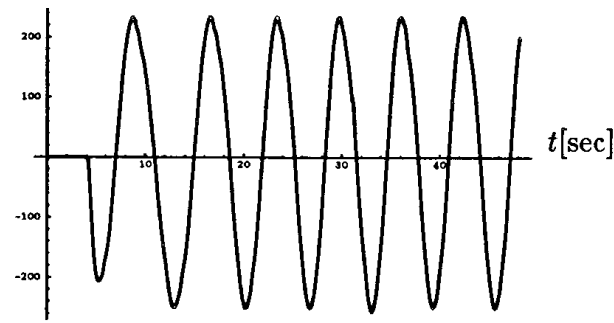

Fig. 15. $Y$ coordinate of the commanded arm control set points for the tracked trajectory as a function of time.

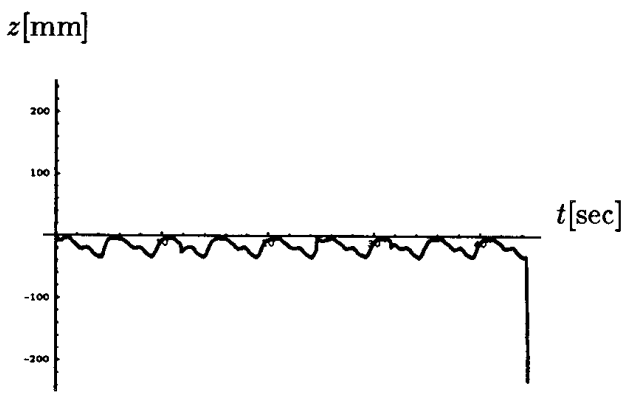

Fig. 16. $Z$ coordinate of the commanded arm control set points for the tracked trajectory as a function of time. The tail is the movement of the arm to effect the grasp of the object.

trajectories in addition to the one shown. The system is also capable of tracking motion direction reversals (with some overshoot) if the train moves forward and then in reverse.

\section{SUMMARY AND FUTURE WORK}

We have developed a robust system for tracking and grasping moving objects. The system relies on real-time stereotriangulation of optic-flow and is able to cope with the inherent noise and inaccuracy of visual sensors by applying parameterized filters that smooth and can predict the moving object's position. Once this tracking is achieved, a grasping strategy is applied that performs an analog of human arm movement schemas.

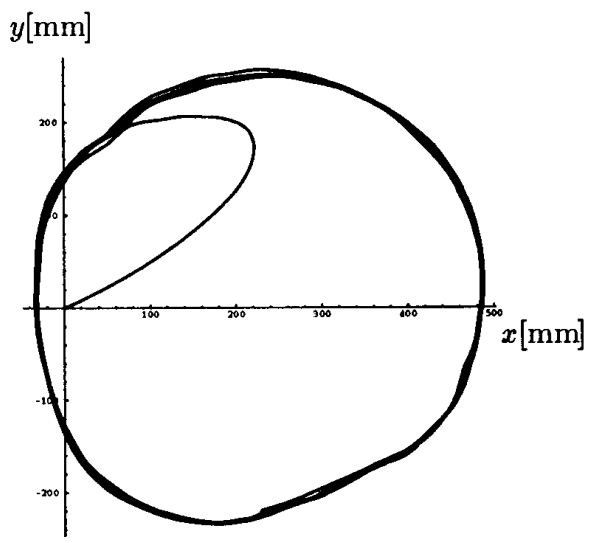

Fig. 17. Trajectory of commanded arm control set points in the $X Y$ plane $X$ and $Y$ are given in millimeters. The train track is an oval, slightly tilted from horizontal axis. The figure shows the system's repeatability over several loops.

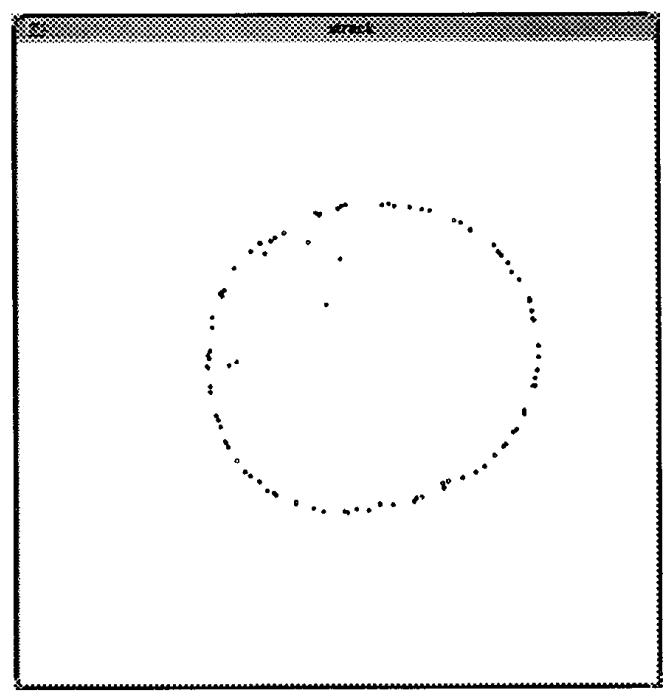

Fig. 18. Object trajectory in $X Y$ plane: on-line graphics window including start-up points.

The system is robust in a number of ways. The vision system does not require special lighting, object structure, or reflectance properties to compute motion since it is based upon calculating optic-flow. The control system is able to cope with the inherent visual sensor noise and triangulation error by using a probabilistic noise model and local parameterization that can be used to build a nonlinear filter to extract accurate control parameters. The arm control system is able to cope with the inherent bandwidth mismatches between the vision sampling rate and the servo-update rate by using a fixedgain predictive filter that allows arm control to function in the occasional absence of a video control signal. Finally, the system is robust enough to repeatedly pick up a moving object and stably grasp it.

We are currently extending this system to other hand-eye coordination tasks. An extension we are pursuing is to im- 


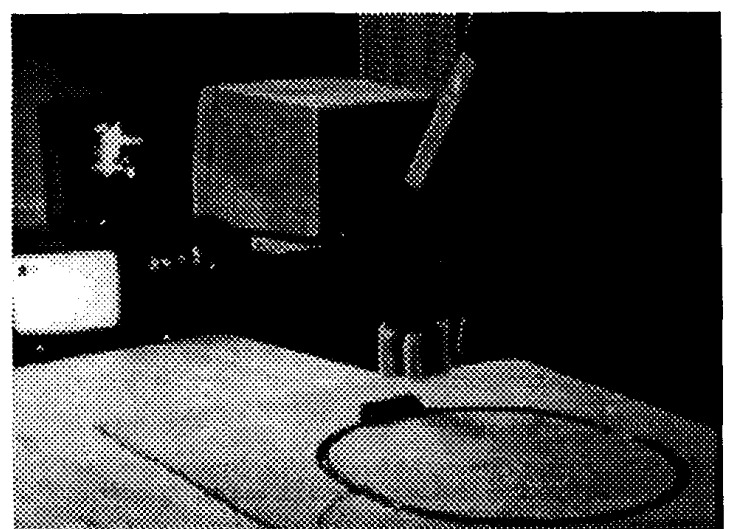

(a)

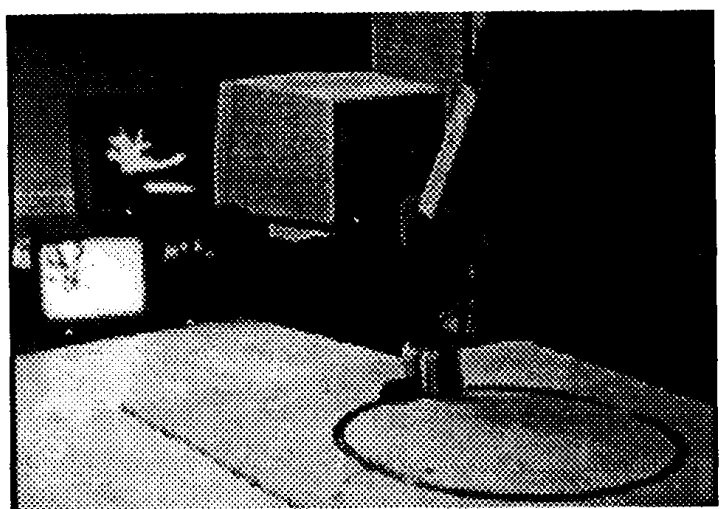

(b)

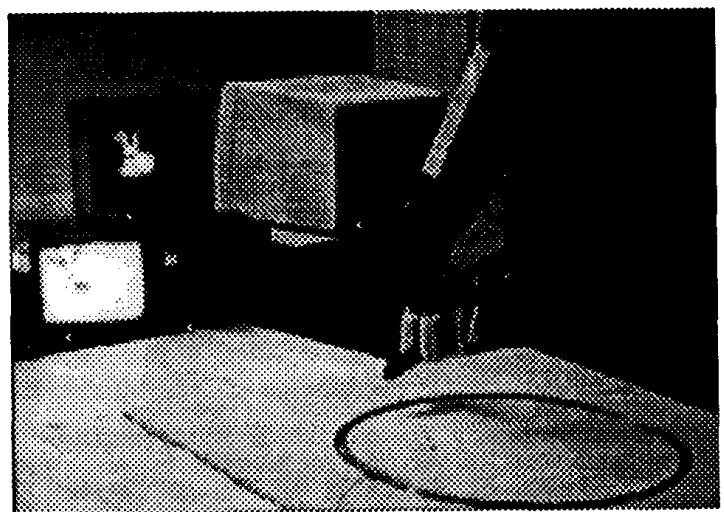

(b)

Fig. 19. Intercepting (a), grasping (b), and picking up (c) the object.

plement other grasping strategies. One strategy is to visually monitor the interception of the hand and object and use this visual information to update the Drive transform at video update rates. This approach is computationally more demanding, requiring multiple moving object tracking capability. The initial vision tracking described above is capable of single object tracking only. If we attempt to visually servo the moving robotic arm with the moving object, we have introduced multiple moving objects into the scene.

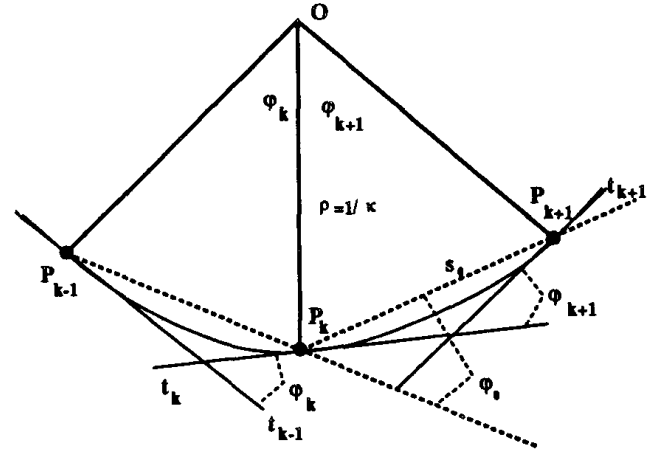

Fig. 20. Trajectory curvature $\kappa$.

We have identified two possible approaches to tracking these multiple objects visually. The first is to use the PIPE's region of interest operator that can effectively "window" the visual field and compute different motion energies in each window concurrently. Each region can be assigned to a different stage of the PIPE and compute its result independently. This approach assumes that the moving objects can be segmented. This is possible since the motion of the hand in 3-D is known-we have commanded it ourselves. Therefore, since we know the camera parameters and 3-D position of the hand, it will be possible to find the relevant image-space coordinates that correspond to the 3-D position of the hand. Once these are known, we can form a window centered on this position in the PIPE, and concurrently compute motion energy of the moving object and the moving hand in each camera. Each of these motion centroids can then be triangulated to find the effective positions of both the hand and object and compute the new Drive transform. Both computations must, however, compete for the hardware histogramming capability needed for centroid computation, and this will effectively reduce the bandwidth of position updating by a factor of 2 .

Another approach is to use a coarse-fine hierarchical control system that uses a multisensor approach. As we approach the object for grasping, we can shift the visual attention from the static cameras used in 3-D triangulation to a single camera mounted on the wrist of the robotic hand. Once we have determined that the moving object is in the field of view of this camera, we can use its estimates of motion via optic-flow to keep the object to grasped in the center of the wrist camera's field of view. This control information will be used to compute the Drive transform to correctly move the hand to intercept the object. We have implemented such a tracking system with a different robotic system [3] and can adapt this method to this particular task.

\section{APPENDIX I \\ TRAJECTORY CURVATURE}

Here we prove that the trajectory curvature is given by the formula $\kappa=\lim _{T \rightarrow 0} \tan \varphi_{0} / s_{0}$ where the following nomenclature is being adopted (see Fig. 20):

- $\kappa$ is the trajectory curvature.

- $T$ is the sampling interval. 
- $P_{k-1}, P_{k}, P_{k+1}$ are three consecutive points along the trajectory.

- $s_{0}=\left\|P_{k+1}-P_{k}\right\|$ is the distance between points $P_{k+1}$ and $P_{k}$.

- $O$ is the center of rotation.

- $\varphi_{k}=\angle P_{k-1} O P_{k}$ is the angle between the tangent lines $t_{k-1}$ and $t_{k}$.

- $\varphi_{k}=\angle P_{k-1} O P_{k}$ is the angle between the tangent lines $t_{k-1}$ and $t_{k}$.

- $\varphi_{0}=\left\{\varphi_{k}+\varphi_{k+1}\right\} / 2$ is the angle between lines $\overline{P_{k-1} P_{k}}$ and $\overline{P_{k} P_{k+1}}$.

- $\psi_{k}$ is the angle which tangent line $t_{k}$ forms with the $x$ axis.

Now we have

$$
\begin{aligned}
\lim _{T \rightarrow 0} & \frac{\tan \varphi_{0}}{s_{0}}=\lim _{T \rightarrow 0} \frac{\frac{\mathrm{d}}{\mathrm{d} T} \tan \varphi_{0}}{\frac{\mathrm{ds}}{\mathrm{d} T}} \\
& =\frac{1}{v} \lim _{T \rightarrow 0} \frac{1}{\cos ^{2} \varphi_{0}} \frac{\mathrm{d}}{\mathrm{d} T} \frac{\psi_{k+1}-\psi_{k-1}}{2} \\
& =\frac{1}{\sqrt{1+y^{\prime 2}}} \lim _{t \rightarrow 0} \frac{\mathrm{d}}{\mathrm{d} T} \frac{\arctan y_{k+1}^{\prime}-\arctan y_{k-1}^{\prime}}{2} \\
& =\frac{y^{\prime \prime}}{\left(1+y^{\prime 2}\right)^{3 / 2}}
\end{aligned}
$$

which is the formula for curvature [14].

\section{APPENDIX II}

\section{VELOCITY EXPECTATION AND VARIANCE}

In order to compute the mathematical expectation of the velocity, we differentiate the following integral (integral 11.4.31 in [1]):

$$
\int_{0}^{\infty} \mathrm{e}^{-a t^{2}} I_{0}(b t) \mathrm{d} t=\frac{1}{2} \sqrt{\frac{\pi}{\alpha}} \mathrm{e}^{b^{2} / 8 a} I_{0}\left(b^{2} / 8 a\right)
$$

with respect to $a$, and by setting $a=1 / 2 \sigma^{2}, b=s_{0} / \sigma^{2}$, we get formula (13).

To prove formula (15) we use formula (11.4.29) from [1] (we set $\nu=0$ ):

$$
\int_{0}^{\infty} \mathrm{e}^{-a t^{2}} t I_{0}(b t) \mathrm{d} t=\frac{\mathrm{e}^{b^{2} / 4 a}}{2 a} .
$$

By differentiation with respect to $a$, and introducing the substitutions for $a$ and $b$ as in (21), we get the formula (15).

\section{APPENDIX III}

\section{VERIFICATION OF FORMULAS (17), (18), AND (19)}

Formula (17) follows from (16) as follows: from $x=$ $\sqrt{p^{2}-2 z^{2}} / z$, we get by solving for $z: z=p / \sqrt{x^{2}+2}$. After substituting the value for $z$ into (16), (17) follows immediately.

Since $x=\sqrt{p^{2}-2 z^{2}} / z=\sqrt{s_{2}^{2}-2 \sigma^{2}} / \sigma$, after solving for $\sigma$ we get $\sigma=s_{2} / \sqrt{x_{0}^{2}+2}$, which is equivalent to (19). From (15) it follows that $s_{0}=\sqrt{s_{2}^{2}-2 \sigma^{2}}$. By substituting the value for $\sigma$, we get $s_{0}=\sqrt{s_{2}^{2}-2 s_{2}^{2} /\left(x_{0}^{2}+2\right)}$. It is easily shown that the last expression is equivalent to (18).

\section{REFERENCES}

[1] M. Abramowitz, Ed., Handbook of Mathematical Functions, National Bureau of Standards, 1964.

[2] E. H. Adelson and J. R. Bergen, "Spatio-temporal energy models for the perception of motion," J. Opt. Soc. Amer., vol. 2, no. 2, pp. 284-299, 1985.

[3] P. Allen, "Real-time motion tracking using spatio-temporal filters," in Proc. DARPA Image Understanding Workshop, Palo Alto, May 1989.

[4] P. K. Allen, B. Yoshimi, and A. Timcenko, "Real-time visual servoing,' in Proc. IEEE Conf. Robotics Automat., 1991, pp. 851-856.

[5] P. Anadan, "Measuring visual motion from image sequences," Tech Rep. COINS TR-87-21, COINS Dep., Univ. Mass., Amherst, 1987.

[6] N. A. Andersen, O. Ravn, and A. T. Sorenson, "Using vision in real-time control systems," in Proc. Amer. Contr. Conf., 1991.

[7] Aspex, PIPE User's Manual.

[8] C. Brown, "Gaze controls with interaction delays," in Proc. DARPA Image Understanding Workshop, May 23-26, 1989, pp. 200-218.

[9] P. J. Burt, J. R. Bergen, R. Hingorani, R. Kolczynski, W. A. Lee, A Leung, J. Lubin, and H. Shvayster, "Object tracking with a moving camera," in Proc. IEEE Workshop Visual Motion, Irvine, CA, Mar. 20-22, 1988, pp. 2-12.

[10] P. J. Burt, C. Yen, and X. Xu, "Multi-resolution flow-through motion analysis," in Proc. IEEE CVPR Conf., 1983, pp. 246-252.

[11] B. F. Buxton and H. Buxton, "Computation of optic flow from the motion of edge features in image sequences," Image Vision Comput., vol. 2, 1984.

[12] J. J. Clark and N. J. Ferrier, "Control of visual attention in mobile robots," in Proc. IEEE Conf. Robotics Automat., May 15-19, 1989, pp. 826-831.

[13] P. Corke, R. Paul, and K. Wohn, "Video-rate visual servoing for sensory-based robotics," Tech. Rep., GRASP Lab., Dep. Comput. Inform. Sci., Univ. Pennsylvania, Philadelphia, 1989.

[14] I. D. Faux and M. J. Pratt, Computational Geometry for Design and Manufacture. New York: Wiley, 1980.

[15] J. T. Feddema and C. S. G. Lee, "Adaptive image feature prediction and control for visual tracking with a hand-eye coordinated camera," IEEE Trans. Syst., Man, Cybern., vol. 20, 1990.

[16] R. Goldenberg, W. C. Lau, A. She, and A. Waxman, "Progress on the prototype pipe," in Proc. IEEE Conf. Robotics Automat., Raleigh, NC. Mar. 31-Apr. 3, 1987

[17] H. H. H. Cruse, J. Dean, and R. Schmidt, "Utilization of sensory information for motor control," in Perspectives on Perception and Action, H. Heuer and A. F. Sanders, Eds. Hillsdale, NJ: Lawrence Erlbaum, 1987, pp. 43-79.

[18] K. Hashimoto, T. Kimoto, T. Ebine, and H. Kimura, "Manipulator control with image-based visual servo," in Proc. IEEE Conf. Robotics Automat., 1991, pp. 2267-2271.

[19] V. Hayward and R. Paul, "Robot manipulator control under UNIX," in Proc. I3th ISIR, Chicago, Apr. 17-21, 1983, pp. 20:32-20:44.

[20] D. Heeger, "A model for extraction of image flow," in lst lnt. Conf. Comput. Vision, London, 1987

[21] B. Hoff and M. Arbib, "Models of trajectory formation and tempora interaction of reach and grasp," Tech. Rep., Center Neural Eng., Univ., Southern Calif., Los Angeles, 1991.

[22] B. K. P. Horn. Robot Vision. Cambridge, MA: M.I.T. Press, 1986.

[23] B. K. P. Horn and B. Schunck, "Determining optical flow," Artif. Intell., vol. 17 , pp. 185-203, 1983

[24] N. Houshangi, "Control of a robotic manipulator to grasp a moving target using vision," in Proc. IEEE Conf. Robotics Automat., 1990.

[25] L. B. Jackson. Digital Filters and Signal Processing. Norwell, MA Kluwer Academic, 1986.

[26] E. W. Kent, M. O. Shneier, and R. Lumia, "Pipe: Pipelined image processing engine," J. Parallel Distributed Comput., vol. 2, pp. 50-78, 1985.

[27] A. J. Koivo and N. Houshangi, "Real-time vision feedback for servoing robotic manipulator with self-tuning controller," IEEE Trans. Syst., Man, Cybern., vol. 21, no. 1, 1991.

[28] S. Lee and Y. Kay, "An accurate estimation of 3D position and orientation of a moving object for robot stereo vision: Kalman filter approach," in Proc. IEEE Conf. Robotics Automat., 1990.

[29] S. W. Lee and K. Wohn, "Tracking moving objects by a mobile camera," Tech. Rep. MS-CIS-88-97, Univ. Pennsylvania, Dep. Comput. Inform. Sci., Philadelphia, Nov. 1988.

[30] R. C. Luo, R. E. Mullen, and D. E. Wessell, "An adaptive robotic tracking system using optical flow," in Proc. IEEE Conf. Robotics Automat., Philadelphia, 1988, pp. 568-573.

[31] W. T. Miller, "Real-time application of neural networks for sensorbased control of robots with vision," IEEE Trans. Syst., Man, Cybern., 
vol. 19, pp. 825-831, July/Aug. 1989

[32] H. H. Nagel, "On the estimation of dense displacement vector fields from image sequences," in Proc. Workshop Motion: Representation and Perception, Toronto, 1983, pp. 59-65.

[33] N. Papanikolopoulos, T. Kanade, and P. Khosla, "Vision and control techniques for robotic visual tracking," in Proc. IEEE Conf. Robotics Automat., 1991, pp. 857-863.

[34] N. Papanikolopoulos, P. K. Khosla, and T. Kanade, "Adaptive robotic visual tracking," in Proc. Amer. Contr. Conf., 1991.

[35] R. Paul. Robot Manipulators. Cambridge, MA: MIT Press, 1981.

[36] B. S. Y. Rao and H. F. Durrant-Whyte, "A fully decentralized algorithm for multi-sensor Kalman filtering," Tech. Rep. OUEL 1787/89, Dep. Eng. Sci., Univ. Oxford, 1989.

[37] R. B. Safadi, "An adaptive algorithm for robotics and computer vision application," Tech. Rep. MS-CIS-88-05, Dep. Comput. Inform. Sci. Univ. Pennsylvania, Jan. 1988

[38] __ "An adaptive tracking algorithm for robotics and computer vision application,"Master's thesis, Univ. Pennsylvania, 1988.

[39] G. L. Scott, "Four-line method of locally estimating optic flow," Image Vision Computing, vol. 5, no. 2, 1986.

[40] A. Singh, "An estimation-theoretic framework for image-flow computation," in Proc. Int. Conf. Comput. Vision (ICCV-90), Kyoto, Japan, Dec. 1990.

[41] G. Verghese, K. G. Lynch, and C. R. Dyer, "Real-time motion tracking of three-dimensional objects," in Proc. IEEE Int. Conf. Robotics Automat. Cincinnati, OH, May 13-18, 1990.

[42] L. E. Weiss, A. Sanderson, and C. P. Neuman, "Dynamic sensor-based control of robots with visual feedback," IEEE J. Robotics Automat. vol. RA-3, pp. 404-417, Oct. 1987.

[43] J. Wiklund and G. Granlund, "Tracking of multiple moving objects," in Time Varying Image Processing and Moving Object Recognition, $\mathrm{V}$. Cappelini, Ed. 1987, pp. 241-249.

[44] M. Xie, "Dynamic vision: Does 3d scene perception necessarily need two cameras or just one?" Tech. Rep., Institut National de Recherche en Informatique et en Automatique, 1989.

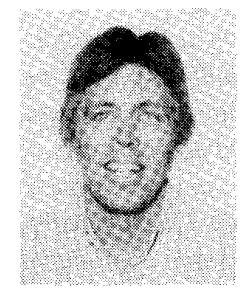

Peter K. Allen ( $\left.\mathrm{S}^{\prime} 82-\mathrm{M}^{\prime} 85\right)$ received the A.B degree from Brown University in MathematicsEconomics, the M.S. degree in computer science from the University of Oregon, and the Ph.D. de gree in computer science from the University of Pennsylvania, where he was the recipient of the CBS Foundation Fellowship, Army Research Office fellowship, and the Rubinoff Award for innovative uses of computers.

He is an Associate Professor of Computer Science at Columbia University and Director of the Center for Research in Intelligent Systems. His current research interests include realtime computer vision, using dextrous robotic hands for object recognition and task-level manipulation, and model-based sensor planning.

Dr. Allen has been named a Presidential Young Investigator by the National Science Foundation. He is a member of ACM and AAAI.

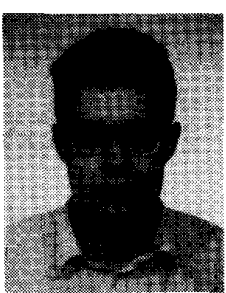

Aleksandar Timcenko (S'90) received the B.S degree in electrical engineering and the M.S. degree in control science from Belgrade University, Yugoslavia, and the M.S. degree in computer science from Columbia University, New York. $\mathrm{He}$ is currently working toward the Ph.D. degree in computer science at Columbia University in the area of motion planning with uncertainties.

His professional interests include modeling and simulation of complex robotic systems.

Billibon Yoshimi (S'89) received both the B.S. and the M.S. degrees in computer science from Columbia University School of Engineering and Applied Science, New York, NY. He is currently working toward the Ph.D. degree in computer science at Columbia University.

His research interest is active visual servoing.

Paul Michelman (S'88-M'92) received the B.A. degree in classics from Grinnell College, Grinnell, IA, the B.E.E.E. degree from the City College of New York, and the M.S. degree in computer science from Columbia University, New York, NY. He is currently working toward the Ph.D. degree in computer science at Columbia University.

His research interests include robotics, tactile sensing, and dextrous manipulation.

Mr. Michelman is a member of Tau Beta Pi. 